\title{
Sweet Basil Seed Mucilage as a Gelling agent in Nasal Drug Delivery
}

\author{
Dhruti Avlani $^{{ }^{*}}$, Dipanjana Ash ${ }^{2}$, Sutapa Biswas Majee ${ }^{2}$ \\ and Gopa Roy Biswas ${ }^{2}$
}

\author{
1 Dey's Medical Stores (Mfg) Ltd. , 62 Bondel Road, Kolkata 700019, India \\ 2 Department of Pharmacy, NSHM Knowledge Campus, Kolkata - Group of \\ Institutions, 124 B.L.Saha Road, Tollygunge, Kolkata 700053, India
}

\begin{abstract}
Intranasal gel formulation with mucilage of natural origin has been potentially explored as an alternative dosage form of drug administration. The purpose of present investigation was to develop paracetamol loaded nasal gel with sweet basil seed mucilage (BSM) as a gelling agent obtained by thermal-hydration process and to characterise both mucilage as well as the gel formulations. Phytochemical screening of BSM reveals it to be rich in carbohydrates. The FT-IR spectrum of the mucilage exhibits the presence of $\mathrm{O}-\mathrm{H}, \mathrm{C}-\mathrm{H}$, and $-\mathrm{COO}^{-}$. The mucilage showed high swelling index of $462 \%$ and relative viscosity of BSM dispersion $(0.25 \% \mathrm{w} / \mathrm{v})$ in nasal medium was found to be 2.94 at $25^{\circ} \mathrm{C}$. Nasal gel formulations demonstrated satisfactory $\mathrm{pH}$, spreadability, extrudibility and drug content. The in vitro release profile of G1 (6\%w/w BSM) demonstrated almost 95\% release with Korsmeyer-Peppas kinetics with highest values of permeability coefficient and steady-state flux. Other formulations with $8-10 \%$ w/w BSM exhibited $70-80 \%$ release within 2.5 hours. G2 and G3 followed zero order kinetics with quasi-Fickian diffusion. The study indicates that G1 $6 \% \mathrm{w} / \mathrm{w}$ BSM) can be selected for nasal gel formulation which may be used for in vivo studies in future. Keywords : Intranasal gel, Mucilage, Quasi-Fickian, Sweet basil seed.
\end{abstract}

\section{Introduction}

Intranasal drug administration is the logical choice for the treatment of local and systemic nasal disorders because of large surface area of nasal mucosa available for drug absorption owing to the presence of numerous microvilli, high vascularization of the subepithelial layer, porous endothelial membrane with a capacity of avoiding hepatic first pass metabolism and thus presents a potentially useful site for the delivery of proteins and peptides. ${ }^{1,2}$ Intranasal administration is non-invasive, painless, does not require a sterile preparation, and the drug can be easily and readily administered in relatively low doses thereby minimizing the systemic toxic effects of oral and parenteral routes. ${ }^{2}$ Examples of commercially available nasally administrated

Dhruti Avlani et al /International Journal of PharmTech Research, 2019,12(3): 42-49.

DOI: http://dx.doi.org/10.20902/IJPTR.2019.120305 
drugs in the form of gels, spray are decongestants for cold symptoms, antihistamines and corticosteroids for rhino sinusitis. ${ }^{3}$ But, muco ciliary clearance, a major disadvantage associated with nasal route can be overcome by increasing the residence time in the nasal cavity through formulation of muco-adhesive in situ gel. ${ }^{4,5}$

In situ hydrogels or prepared hydrogel-based formulations allow intimate contact with the mucosal surface, prolong the residence time and improve drug bioavailability over liquid preparations. Hydrogels of different polymers vary in their pore size, rate of degradation, mechanical strength and drug release mechanism. Drug release can be tailor-made according to the therapeutic demands and can be suitably controlled by altering the nature and composition of hydrogel formulations and hence, their physicochemical and pharmaceutical properties. $^{6,7}$

The main objective of present investigation was to formulate paracetamol loaded nasal gel by using mucilage extracted from Ocimum basilicum L. seeds as a gelling agent and to characterise both mucilage as well as the gel formulations.

\section{Materials and method}

\section{Materials}

Sweet basil seed i.e. Ocimum basilicum L. seeds used for mucilage extraction were purchased from the local market, Kolkata, West Bengal, India. The seeds were cleaned and stored in air tight containers until further use. Identification and authentication of the Ocimum basilicum L. plant specimen D-C1 was done at Central National Herbarium, Botanical Survey of India, Shibpur, Howrah. All reagents used in the investigation were of analytical grade and were purchased from Merck Specialities Pvt. Ltd. or prepared from the raw materials in the laboratory according to standard procedures (as for phytochemical reagents). For rheological measurements and swelling study in nasal medium, sodium carboxy methyl cellulose (Na-CMC) and psyllium were employed as the control respectively.

\section{Extraction of Ocimum basilicum L. seed mucilage}

Basil seed mucilage was obtained by thermal-hydration process. The whole nutlets were soaked in hot distilled water, in a seed: water ratio of 1:50. The mucilage of basil seeds was extracted by continuous stirring on a mechanical stirrer at $1500 \mathrm{rpm}$ for $4 \mathrm{~h}$ at $40^{\circ} \mathrm{C}$. Vacuum filtration was carried out to remove all likely seed residuals from the separated mucilage. Pure ethanol was added to the extracted mucilage (3:1) and left overnight at $4^{\circ} \mathrm{C}$ for removal of protein and ash content. Crude extract was concentrated at $55^{\circ} \mathrm{C}$ with rotary vacuum evaporator (REMI Instruments Ltd.) to remove extra water/ethanol content and then dried on stainless steel trays in a laboratory oven at $50^{\circ} \mathrm{C}$ for $6 \mathrm{~h}$ to produce dry basil seed mucilage (BSM). ${ }^{8}$ The dried mucilage was ground into powder to pass $200 \mu \mathrm{m}$ sieves. The mucilage powder was stored in desiccator at a temperature of $30^{\circ} \mathrm{C}$ and $75 \% \mathrm{RH}$ for further studies. ${ }^{9}$

\section{Characterisation of seed mucilage}

\section{Chemical characterisation}

\section{Fourier-transformed Infra-red (FTIR) spectroscopy}

FTIR spectroscopy was carried out in order to assign functional groups to the isolated mucilage. ${ }^{10}$ For sample preparation, the samples were powdered as finely as possible to minimise IR scattering on the particle surface and the portion of the reflected light and pellets were prepared using potassium bromide. The potassium bromide-sample pellets were observed in the FTIR spectrometer (Bruker, Alpha-T, Germany) in the range of $4000-400 \mathrm{~cm}^{-1}$.

\section{Phytochemical test}

Dried and powdered BSM was analyzed for the presence of various phytoconstituents such as carbohydrates, alkaloids, phenols, flavonoids, saponins, tannins, steroids, glycosides based on the standard protocols. ${ }^{11}$ 


\section{Physical characterisation}

\section{Water-Holding Capacity (WHC)}

For estimation of WHC, dried $0.1 \mathrm{~g}$ sample was weighed and then stirred into $20 \mathrm{~mL}$ of distilled water for $1 \mathrm{~min}$. The corresponding mucilage dispersions were then centrifuged (REMI Test Master) at $2200 \mathrm{X} \mathrm{g}$ for $30 \mathrm{~min}$ and the weight of supernatant was measured. Water-holding capacity was expressed as $\mathrm{g}$ of water held per $g$ of sample. ${ }^{12}$

\section{Swelling study}

Nasal medium was prepared using $0.65 \% \mathrm{NaCl}, \quad 0.04 \% \quad \mathrm{KH}_{2} \mathrm{PO}_{4}, \quad 0.09 \% \quad \mathrm{~K}_{2} \mathrm{HPO}_{4}$ and $0.02 \%$ benzalkonium chloride (pH 6) ${ }^{13}$ Mucilage dispersion in nasal medium was prepared at a concentration of 0.25 $\% \mathrm{w} / \mathrm{v}$. It was then left undisturbed for $6 \mathrm{~h}$ at $25^{\circ} \mathrm{C}$. The volume occupied by mucilage was measured every hour and at $6^{\text {th }}$ hour, the supernatant was decanted and the volume of the final swollen gel was recorded. The swelling index was calculated as follows ${ }^{9}$ :

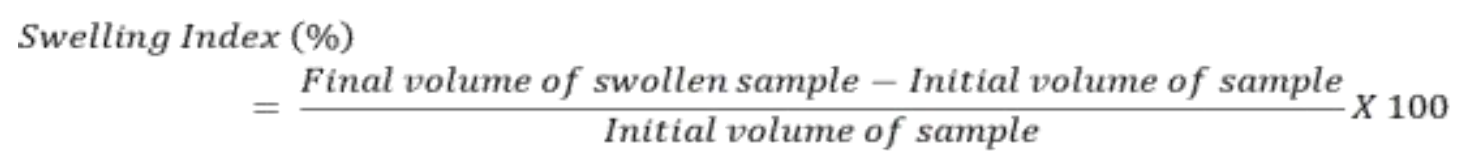

\section{Rheological study}

BSM dispersion $(0.25 \% \mathrm{w} / \mathrm{v})$ was prepared in nasal medium using a mechanical stirrer at $40^{\circ} \mathrm{C}$. After cooling, the dispersion was left overnight at $4^{\circ} \mathrm{C}$ to ensure complete hydration prior to the rheological measurements. The viscosity of BSM dispersions was determined using Ostwald viscometer at $25^{\circ} \mathrm{C}$ by employing the following equation:

Relative Viscosity $=\eta_{\text {rel-mucilage,conc }}=\frac{\rho_{\text {mucilage }} t_{\text {mucilage }}}{\rho_{\text {water }} t_{\text {water }}}$

\section{Gel clarity}

Mucilage dispersions $(0.0625-1.0 \% \mathrm{w} / \mathrm{w})$ in nasal medium were prepared and centrifuged at $6000 \mathrm{rpm}$ for 15 mins. Clarity of the supernatant was measured spectrophotometrically (UV-VIS Spectrophotometer, Shimadzu Cooperation, UV-1800) at $650 \mathrm{~nm}^{14}$

\section{Formulation and characterisation of nasal gels}

\section{Gel formulation}

Four different batches of nasal gel (30gms) containing model drug, paracetamol (PCM) $(1 \% \mathrm{w} / \mathrm{w})$ were prepared using BSM as gelling agent in the concentration of $6,8,10$ and $12 \% \mathrm{w} / \mathrm{w}$ in nasal medium. The prepared gels were transferred in a collapsible tube, crimped and labelled as G1 (6\%w/w), G2 (8\%w/w), G3 $(10 \% \mathrm{w} / \mathrm{w})$ and $\mathrm{G} 4(12 \% \mathrm{w} / \mathrm{w})$.

\section{Characterisation of gels}

The prepared gels were evaluated for extrudability $(\mathrm{cm})$, spreadability $\left(\mathrm{g} \mathrm{cm} \mathrm{sec}^{-1}\right)$ and percent drug content. Extrudability is the distance travelled in $\mathrm{cm}$ by a ribbon of gel in 10 seconds during extrusion from the collapsible tube and is expressed as $\mathrm{cm} / \mathrm{sec}$. For the determination of spreadability, excess sample was applied between two glass slides and was compressed to uniform thickness. Spreadability was calculated by using the equation given below 
Where, $\mathrm{M}$ is the weight tied to upper slides $(20 \mathrm{~g}), \mathrm{L}$ is the length of the glass slide $(7.5 \mathrm{~cm})$ and $\mathrm{T}$ is the time taken in sec. ${ }^{15}$

\section{Determination of drug content}

Drug content of gels was determined by standard methods. ${ }^{15,16}$

\section{Characterisation and comparison of in vitro drug diffusion profiles of nasal gels}

Paracetamol diffusion from nasal gels was studied in nasal medium through synthetic membrane (Hi Media Laboratory Pvt. Ltd.) in modified Franz diffusion cell at $34 \pm 0.1^{\circ} \mathrm{C}$ for $6 \mathrm{hr}$. Aliquots were measured at pre-determined time points and absorbances measured spectrophotometrically at $242 \mathrm{~nm} .{ }^{17}$ Percentage of drug release was calculated from calibration curve of paracetamol in nasal medium. Model-dependent method was adopted for comparison of drug diffusion profiles of the gel formulations. The drug diffusion data were fitted to four different kinetic mathematical models namely, zero order, first order, Higuchi and Korsmeyer-Peppas release equations to find out the best suitable model from comparison of $\mathrm{R}^{2}$ values. ${ }^{18}$

The steady-state flux $\left(\mathrm{SS}_{\text {flux }}\right)$ of drug from gels across the membrane is defined as follows

SSflux $=\frac{d Q}{d t} X \frac{1}{A}$

Where, $\mathrm{dQ} / \mathrm{dt}$ is the slope of the linear portion of the curve i.e. cumulative amount per unit time $(\mathrm{mg} / \mathrm{min})$ and $\mathrm{A}$ is the diffusional area $(\mathrm{sq} \mathrm{cm})$. The measurement of flux across human skin provides a valuable insight into the formulation development of any dermatological product. For the quantification of permeability coefficient, $K_{p}$, the following equation is employed

$$
\text { SSflux }=K_{p} X C_{a p p}
$$

Where, $\mathrm{C}_{\text {app }}$ is the initial concentration of the drug $(\% \mathrm{w} / \mathrm{w})$ in the gel. ${ }^{15}$

\section{Statistical analysis:}

Data have been obtained from each experiment in triplicate $(n=3)$ and were subjected to statistical analysis using one way analysis of variance (ANOVA). Results are quoted as significant where $p<0.05$.

\section{Results and discussion}

The yield of the mucilage from the basil seeds was approximately $20-25 \%$ of dry seed mass. In the literature, yields of 7.86-25\% have been reported. ${ }^{19,20}$

\section{Chemical characterisation of seed mucilage}

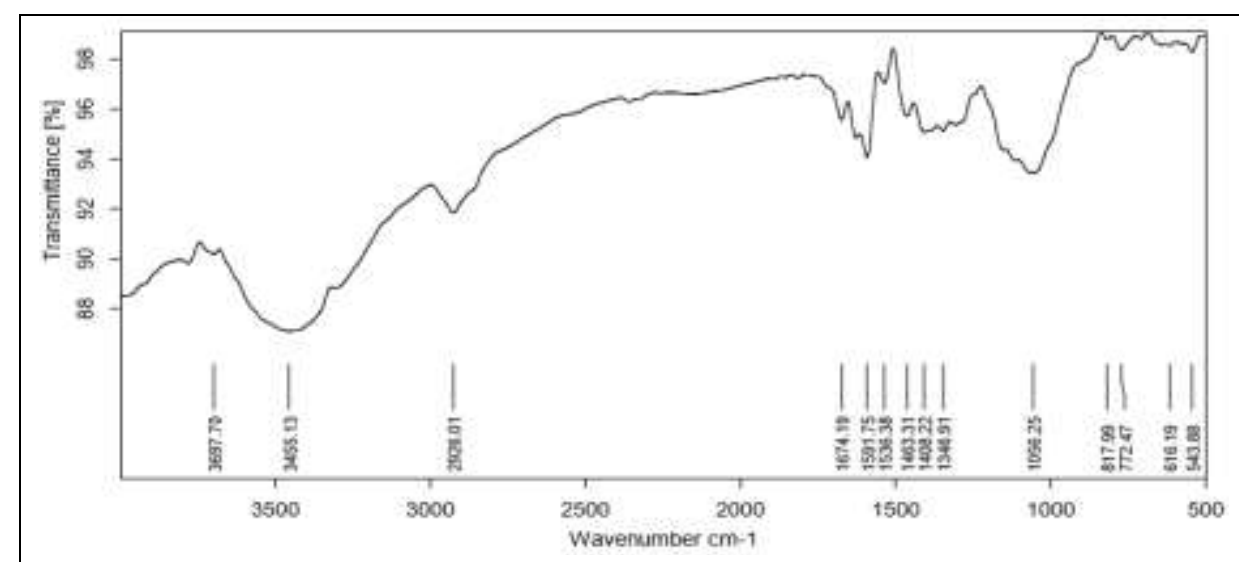

Fig 1: FTIR spectrum of sweet basil seed mucilage (BSM) 
FTIR spectrum (fig.1) of BSM shows characteristic bands at approximately 3455.13, 2926.01 and $1674.19 \mathrm{~cm}^{-1}$, which are found in polysaccharides and represent hydroxyl (-OH) stretching, $\mathrm{C}-\mathrm{H}$ stretching of the $\mathrm{CH}_{2}$ groups, and $-\mathrm{COO}^{-}$(asymmetric vibrations) groups, respectively, in carbohydrate and uronic acid molecules. The peaks are similar to those observed in xanthan gum and guar gum. ${ }^{21}$ In a previous study, the peak at $1589 \mathrm{~cm}^{-1}$ observed for basil seed mucilage was attributed to $\mathrm{N}-\mathrm{H}$ primary amide. It was suggested that the basil seed mucilage is neither starch nor cellulosic polysaccharide, but possesses peptide cross-links and amino sugars. ${ }^{8}$ The absorption band at $1674.19 \mathrm{~cm}^{-1}$ may be attributed to ring stretching of mannose as has been reported for locust bean gum and guar gum. ${ }^{22}$

Preliminary phytochemical screening of Ocimum basilicum L. seed mucilage isolated in the present study revealed the presence of non-reducing sugars, proteins, gums and mucilage and confirmed the absence of alkaloid, tannins and saponins.

\section{Physical characterisation of seed mucilage}

The water-holding capacity of mucilage from sweet basil seeds has been found to be $97.5 \pm 2.4 \mathrm{~g} / \mathrm{g}$ mucilage which is close to the reported value of $103.2 \mathrm{~g}$ water/g fiber for fatted chia gum. ${ }^{12}$ Thus, the mucilage is expected to demonstrate high swelling index and form a gel on hydration. The mucilage indeed showed high swelling index of $462 \%$ and relative viscosity of BSM dispersion $(0.25 \% \mathrm{w} / \mathrm{v})$ in nasal medium was found to be 2.94 at $25^{\circ} \mathrm{C}$.

\section{Preparation and characterisation of nasal gels}

\section{Gel clarity}

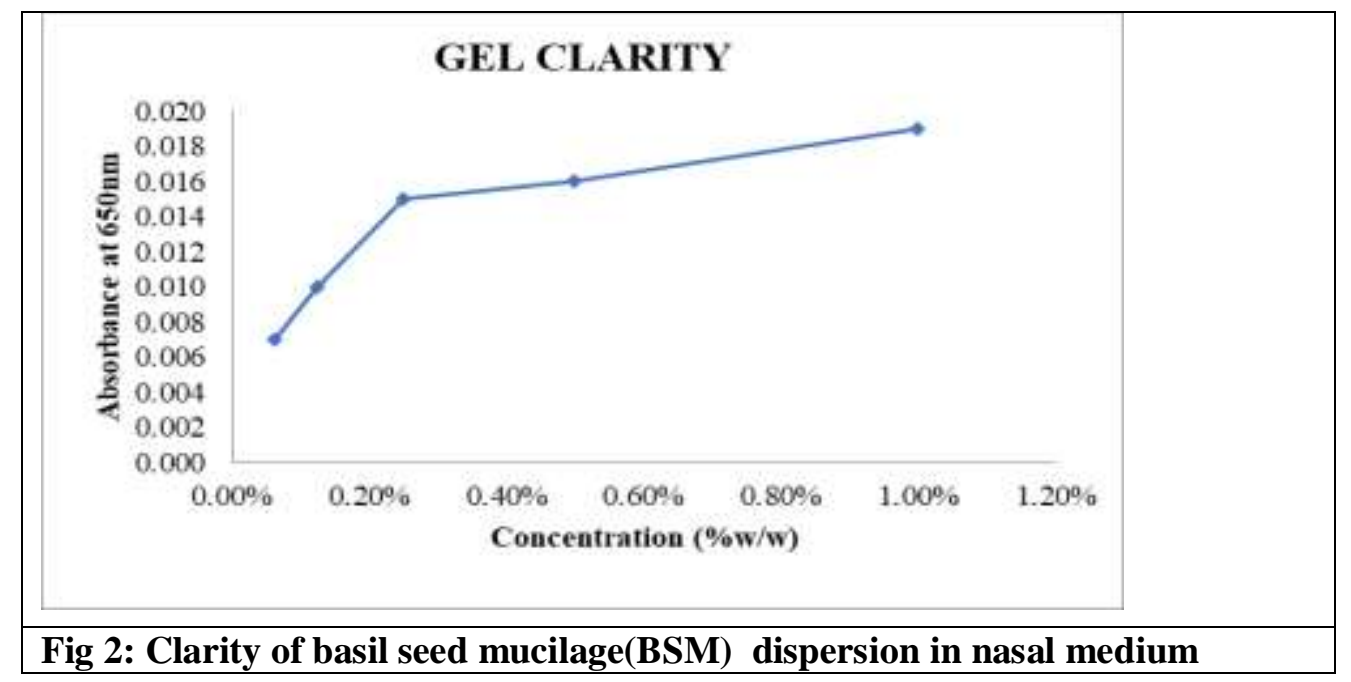

Degree of swelling of the mucilage directly influences gel clarity. Dispersions containing higher percentage of BSM showed lower clarity (fig. 2). The clarity of the BSM based gel may directly influence the shine of products that contain it as a gelling agent. ${ }^{14}$

\section{As Gelling Agent}

Evaluation parameters for nasal gel formulation containing BSM as gelling agent $(6-12 \% \mathrm{w} / \mathrm{w})$ are provided in Table 1. The gel formulations were creamish white in colour, smooth to feel and opaque in nature. 
Table 1: Nasal gel formulations using BSM and their evaluation parameters. Data represent mean \pm standard deviation for 3 experiments $(n=3)$

\begin{tabular}{|c|c|c|c|c|c|c|}
\hline $\begin{array}{l}\text { Batch } \\
\text { No. }\end{array}$ & $\begin{array}{l}\% \text { BSM } \\
(\% \mathrm{w} / \mathrm{w})\end{array}$ & $\begin{array}{l}\text { Net Content } \\
\text { (gm) }\end{array}$ & $\mathbf{p H}$ & $\begin{array}{l}\text { Spreadability } \\
\left(\mathrm{g} \mathrm{cm} \mathrm{sec}^{-1}\right)\end{array}$ & $\begin{array}{l}\text { Extrudability } \\
\text { (cm/sec) }\end{array}$ & $\begin{array}{l}\text { Percent } \\
\text { Drug } \\
\text { Content }\end{array}$ \\
\hline G1 & 6 & 30 & $6.0 \pm 0.5$ & $1.50 \pm 0.7$ & $0.75 \pm 0.6$ & $99 \pm 0.2$ \\
\hline G2 & 8 & 30 & $6.0 \pm 0.4$ & $1.30 \pm 0.6$ & $0.8 \pm 0.2$ & $97 \pm 0.3$ \\
\hline G3 & 10 & 30 & $6.0 \pm 0.2$ & $1.26 \pm 0.5$ & $0.9 \pm 0.3$ & $95 \pm 0.6$ \\
\hline G4 & 12 & 30 & $6.0 \pm 0.3$ & $0.5 \pm 0.5$ & $0.5 \pm 0.6$ & $90 \pm 0.2$ \\
\hline
\end{tabular}

Table 2: Drug diffusion parameters of paracetamol-loaded nasal gels with BSM as gelling agent. Data represent mean \pm standard deviation for 3 experiments $(n=3)$

\begin{tabular}{|l|l|l|l|l|}
\hline Batch No. & \% BSM (\% w/w) & $\mathbf{t}_{\mathbf{5 0}}(\mathbf{h r s})$ & $\mathbf{S S}_{\text {flux }}(\mathbf{m g . c m} / \mathbf{m i n})$ & $\mathbf{K}_{\mathbf{p}}\left(\mathbf{c m}^{2} / \mathbf{m i n}\right)$ \\
\hline G1 & 6 & $<0.5 \pm 0.2$ & $14.902 \pm 0.2$ & $14.902 \pm 0.8$ \\
\hline G2 & 8 & $0.72 \pm 0.3$ & $10.920 \pm 0.4$ & $10.920 \pm 0.2$ \\
\hline G3 & 10 & $0.68 \pm 0.5$ & $12.398 \pm 0.6$ & $12.398 \pm 0.6$ \\
\hline G4 & 12 & $1.43 \pm 0.6$ & $10.030 \pm 0.7$ & $10.030 \pm 0.3$ \\
\hline
\end{tabular}

In vitro drug diffusion profiles of G1 to G4 are presented in Table 2. The in vitro release profile of G1 $(6 \% \mathrm{w} / \mathrm{w}$ BSM) demonstrated almost $95 \%$ release (fig.3). For the other three formulations containing $8 \%, 10 \%$ and $12 \% \mathrm{w} / \mathrm{w}$ BSM, only $70-80 \%$ release could be achieved within 2.5 hours. No further drug release was observed during the remaining 4 hours of the diffusion study. Apparent permeability coefficients were calculated as per the equation 5. Permeability coefficient of the gel containing $6 \% \mathrm{w} / \mathrm{w} \mathrm{BSM}$ was found to be the highest and the $\mathrm{SS}_{\text {flux }}$ for the above formulation was $14.902 \pm 0.2 \mathrm{mg} . \mathrm{cm} / \mathrm{min}$.

Table 3. Kinetic modelling of drug release data from drug-loaded nasal gels with BSM

\begin{tabular}{|l|l|l|l|}
\hline Batch & Kinetics followed & 'n' value & Type of diffusion \\
\hline G1 & Korsmeyer-Peppas & 0.348 & Quasi-Fickian \\
\hline G2 & Zero-order & 0.298 & Quasi-Fickian \\
\hline G3 & Korsmeyer-Peppas & 0.351 & Quasi-Fickian \\
\hline G4 & Zero-order & 0.329 & Quasi-Fickian \\
\hline
\end{tabular}

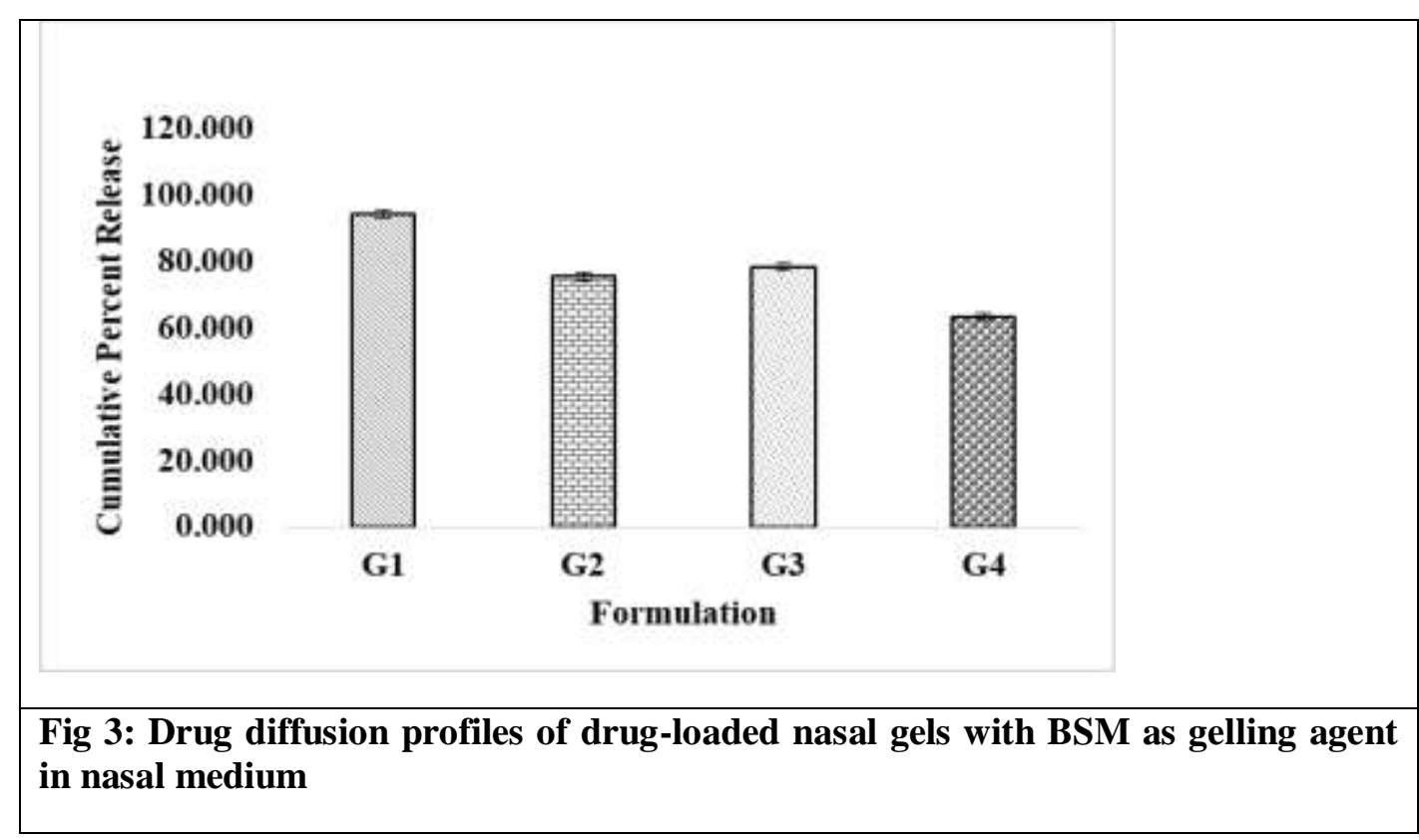


Based on the analysis of release kinetics, it has been observed that the nasal gels containing 8 and 12 $\% \mathrm{w} / \mathrm{w}$ BSM followed zero order kinetics, whereas for the gels containing 6 and $10 \% \mathrm{w} / \mathrm{w}$ BSM, KorsmeyerPeppas model was followed. In all cases, quasi-Fickian diffusion was observed. ${ }^{23}$ Thus from the above results, G1 (6 \% w/w BSM) can be selected for in vivo studies in future, since it demonstrated maximum percentage of drug diffusion from the gel across the synthetic membrane and can exhibit sustained and complete drug release.

\section{Conclusion}

The studies on the mucilage obtained from the seed of Ocimum basilicum L. demonstrated its gelling property and swellability and its potential for use in nasal gel formulations, owing to its hydrophilic nature, physical stability, extrudability, good spreadability and good drug release profile.

\section{Acknowledgement}

The author(s) would like to acknowledge Department of Pharmacy, NSHM Knowledge Campus for providing an institutional research platform and necessary facilities.

\section{References}

1. Upadhyay S, Parikh A, Joshi P, Upadhyay UM and Chotai N.P. Intranasal drug delivery system-A glimpse to become maestro, J App Pharm Sci, 2011, 1, 34-44.

2. Appasaheb PS, Manohar SD and Bhanudas SR. A review on intranasal drug delivery system, J Adv Pharm Edu Res, 2013, 3, 333-46.

3. Marx D, Williams $G$ and Birkhoff M. Intranasal drug administration- an attractive delivery route for some drugs, Drug discovery and development-From molecules to medicine, IntechOpen, Croatia, 2015, 299-320. doi: 10.5772/59468.

4. Delyle SG, Buenestado A, Naline E, Faisy C, Laye SB, Couderc LJ, Guen ML, Fischler M and Devillier P. Intranasal drug delivery: An effective and non-invasive route for systemic administration focus on opioids, Pharmacol Ther, 2012, 134, 366-79.

5. Wang Y, Jiang S, Wang $\mathrm{H}$ and Bie $\mathrm{H}$. A mucoadhesive, thrmoreversible in situ nasal gel of geniposide for neurodegenerative diseases, PLoS One, 2017, 17 pgs. doi:10.1371/ Journal. pone 0189478.

6. Nazar H, Fatouros DG, Vandermerwe SM, Bouropoulos N, Avgouropoulos G, Tsibouklis J and Roldo $\mathrm{M}$. Thermosensitive hydrogels for nasal drug delivery: The formulation and characterisation of systems based on N-trimethyl chitosan chloride, Eur J Phar Biopharm, 2011,77, 225-32.

7. Luppi B, Bigucci F, Cerchiara T and Zecchi V. Chitosan-based hydrogels for nasal drug delivery: From inserts to nanoparticles, Expert Opin Drug Deliv, 2010, 7, 811-28.

8. Akbari I and Ghoreishi SM. Generation of porous structure from basil seed mucilage via supercritical fluid assisted process for biomedical applications, Int J Pharm Sci Dev Res, 2017, 3, 30-5.

9. Munoz LA, Cobos A, Diaz O and Aguilera JM. Chia seeds: Microstructure, mucilage extraction and hydration, J Food Eng, 2012, 108, 216-24.

10. Timilsena YP, Adhikari R, Kasapis S, and Adhikari B. Molecular and functional characteristics of purified gum from Australian chia seeds, Carb Poly, 2016, 136, 128-36.

11. Kassakul W, Praznik W, Viernstein H, Phrutivora P and Leela P. Characterisation of the mucilages extracted from Hibiscus rosa-sinensis Linn and Hibiscus mutabilis Linn and their skin moisturizing effect, Int J Pharm Pharm Sci, 2014, 6, 453-7.

12. Campos MRS, Solis NC, Rubio GR, Chel GL and Betancur AD. Chemical and functional properties of chia seed gum, Int J Food Sci, 2014, 5 pages. http://dx.doi.org/10.1155/2014/241053.

13. Basu S, Chakraborty A and Bandyopadhyay AK. Development and evaluation of a mucoadhesive nasal gel of midazolam prepared with Linum usitatissimum L. seed mucilage, Scientia Pharmaceutica, 2009, 77,899-910.

14. Ancona D, Lopez LJ and Chel GL. Comparison of the chemical composition and functional properties of Phaseolus lunatus prime and tailing starches, Food Chem, 2003, 82, 217-25.

15. Aiyalu R, Govindarjan A and Ramasamy A. Formulation and evaluation of topical herbal gel for the treatment of arthritis in animal model, Braz J Pharm Sci, 2016, 52, 493-507. 
16. Indian Pharmacopoeia, Government of India, Ministry of Health and Family Welfare Department, 7th edition, 2014, 1, 559-621.

17. Indian Pharmacopoeia, Government of India, Ministry of Health and Family Welfare Department, $7^{\text {th }}$ edition, 2014, 1, 187-94.

18. Costa P and Lobo JMS. Modeling and comparison of dissolution profiles, Eur J Pharm Sci, 2001, 13, 123-33.

19. Nazir S, Wani I and Masoodi FA. Extraction optimization of mucilage from basil (Ocimum basilicum L.) seeds using response surface methodology, J Adv Res, 2017, 8, 235-44.

20. Akbari I and Ghoreishi SM. Generation of porous structure from basil seed mucilage via supercritical fluid assisted process for biomedical applications, Int J Pharm Sci Dev. Res., 2017, 3, 30-5.

21. Goh KKT, Merino L, Chiang JH, Quek R, Soh SJB and Lentle RG. The physico-chemical properties of chia seed polysaccharide and its microgel dispersion rheology, Carb Polym, 2016,149, 297-307.

22. Monrroy M, Garcia E, Rios K, Garcia JR. Extraction and physicochemical characterization of mucilage from Opuntia cochenillifera (L.) Miller, J Chem, 2017, 9 pages. https://doi.org/10.1155/2017/4301901.

23. Rosun $\mathrm{MC}$ and Bratu I, Promising psyllium-based composite containing $\mathrm{TiO}_{2}$ nanoparticles as aspirincarrier matrix, Progr Natur Sci Mat Int, 2014, 24, 205-09. 